\title{
Fragmentation of $\mathrm{H}_{2}$-molecules studied with laser-induced Coulomb explosion imaging and femtosecond pump-probe experiments
}

\author{
Th. Ergler*, A. Rudenko, B. Feuerstein, K. Zrost, C.D. Schröter,R. Moshammer and J. Ullrich \\ Max-Planck-Institut für Kernphysik, D-69029 Heidelberg, Germany
}

\begin{abstract}
We report on the experimental realisation of time-resolved coincident Coulomb explosion imaging of $\mathrm{H}_{2}$-fragmentation in $10^{14} \mathrm{~W} / \mathrm{cm}^{2}$ laser fields. Combining a high-resolution 'reaction microscope' and a fs pump-probe setup, we map the motion of wave packets dissociating via one- or two-photon channels, respectively, and observe two region of enhanced ionization in accordance with earlier theoretical predictions. The long-term interferometric stability of our system allows us to extend pump-probe experiments into the region of overlapping pulses, which offers new possibilities for the manipulation of ultrafast molecular fragmentation dynamics.
\end{abstract}

Keywords: pump-probe experiments, reaction microscope, coincident measurements, Coulomb explosion, dissociation.

\section{INTRODUCTION}

Molecular dynamics in strong laser fields has recently attracted increasing attention experimentally as well as theoretically ${ }^{1,2}$, fuelled not at least by the tremendous progress achieved in ultrafast laser technology. State-of-the-art infrared lasers routinely provide pulses shorter than $30 \mathrm{fs}$, which match the vibrational timescale for most molecular systems. This offers a variety of possibilities to study molecular motion in different physical, chemical or biological processes $^{3}$. Most of these experiments are based on the so-called pump-probe scheme, where the first (pump) laser pulse initiates a particular molecular reaction, whereas the second (probe) pulse, which arrives after a certain time delay, is used to trace the following time-dependent dynamics of the process of interest. In order to explore the time evolution of the molecular system, the probe stage may exploit different laser-induced phenomena like absorption and scattering of light, fluorescence or photoionization. In the latter case, if two or more electrons are removed, the molecule can explode due to a Coulomb repulsion. The measurement of (angle-resolved) kinetic energy spectra of ionic fragments provides information about the molecular structure at the moment of explosion. This technique, known as Coulomb explosion (CE) imaging, was originally developed for fragmentation of fast molecular beams passing thin foils ${ }^{4,5}$. Combined with an fs pump-probe scheme, it provides one of the most direct methods of mapping both, the structure and the dynamics of moving nuclear wave packets of molecules. Here, the pump laser pulse prepares a molecular wave packet (e.g., initiates a dissociative ionization), and the probe pulse projects the wave function onto the repulsive Coulomb potential curve. The wave packet shape is then reconstructed from the measured kinetic energy distribution of the reaction fragments using the Coulomb law. Being first applied to study the dissociation of $\mathrm{I}_{2}$ molecules ${ }^{6,7}$, this method was later extended to the simplest (and fastest) diatomic systems, such as $\mathrm{H}_{2}{ }^{8}$ or $\mathrm{D}_{2}{ }^{9}$. The latter are the ideal candidates for testing the $\mathrm{CE}$ imaging schemes for two reasons. First, after the removal of both electrons they represent a pure twocenter Coulomb system. Second, their fragmentation in intense laser fields was extensively studied experimentally and theoretically ${ }^{1-2}$.

There are two basic requirements for the applicability of laser-induced CE imaging. First, one has to provide a reliable way to distinguish CE fragments from other (e.g., dissociated) reaction products. This, in case of $\mathrm{H}_{2}\left(\mathrm{D}_{2}\right)$, requires coincident measurement of both protons. Otherwise, the CE mapping of molecular dissociation for these targets is either restricted to the regions of small internuclear separations $\mathrm{R}$, where the Coulomb energy clearly differs from the energy of the dissociated fragments ${ }^{9}$, or it requires the use of two pulses with different polarization ${ }^{8}$. Second, the effective duration of a probe pulse should be shorter than the time scale of the process of interest. Thus, in order to resolve the motion of nuclear wave packets of bound molecules, laser pulses shorter than the vibrational period are required, which

\footnotetext{
*t.ergler@mpi-hd.mpg.de phone: +496221516296 fax: +496221516604
} 
for the case of $\mathrm{H}_{2}\left(\mathrm{D}_{2}\right)$ would mean sub-10 fs pulses. An alternative method suggests using electrons which return back to their parent ion and can induce a second ionization step as a probe pulse (the so called molecular clock scheme) ${ }^{10,11}$. Providing a sub-laser cycle time resolution, this method is restricted by fixed return times and by the difficulties in separating CE events induced by recollision from other field-induced processes. For the case of dissociating molecules the duration of a probe pulse limits the ultimate spatio-temporal resolution of the experiment. However, as it was pointed out by Stapelfeldt et $a l^{7}$, the effective pulse duration, where the second ionization step takes place, is only a fraction of the full pulse length, provided the laser intensity is kept below the saturation value. Thus, the real experimental resolution is often limited not only by the probe pulse duration but by the resolution of the energy measurement as well as.

In this paper we report on the experimental realisation of time-resolved coincident Coulomb explosion imaging of dissociating $\mathrm{H}_{2}$ molecules. Using a combination of the "reaction microscope" spectrometer, which allows coincident detection of several charged particles with excellent momentum resolution, and a pump-probe setup providing two identical 25 fs pulses with variable delay at sub-fs accuracy, we are able to resolve in space and time the motion of two different dissociating wave packets produced by net absorption of one or two photons. We provide experimental evidences of the existence of a second maximum in the R-dependent ionization probability for $\mathrm{H}_{2}^{+}$, which was predicted in several previous theoretical studies ${ }^{12-15}$. Finally, we demonstrate that by using an interferometrically stable pumpprobe setup which allows control of the electric field shape in the region of overlapping pulses, a clear pump probe experiment can be carried out even in this regime by selecting only those delays for which both pulses interfere destructively. Moreover, this simplest form of pulse-shaping allows us to manipulate the ultrafast molecular fragmentation dynamics, suppressing and enhancing certain fragmentation channels.

The paper is structured as follows: In Section 2 a description of the experimental set-up is given, followed by a brief overview of the basic fragmentation channels of $\mathrm{H}_{2}$ in intense laser fields in Section 3. The experimental results are presented and discussed in Section 4, and conclusions are given in Section 5. Atomic units (a.u.) are used if not stated otherwise.

\section{EXPERIMENTAL SETUP}

The sketch of the experimental setup is shown in Fig. 1. Measurements were performed using linearly polarized radiation from a Kerr-lens mode locked Ti:sapphire laser at $795 \mathrm{~nm}$ central wavelength with 25 fs pulse width (FWHM) and $3 \mathrm{kHz}$ repetition rate. The laser beam was fed through a Mach-Zehnder type interferometer with one arm variable in length, providing two identical pulses separated by a time delay which can be scanned from 0 to 3300 fs with a resolution of better than 300 as. Both laser beams were focused to a spot size of $\sim 5 \mu \mathrm{m}$ onto the collimated supersonic molecular gas jet in the center of an ultra-high vacuum chamber $\left(2 \cdot 10^{-11} \mathrm{mbar}\right)$. Charged reaction fragments were guided to two position-sensitive channel plate detectors by weak electric $(2 \mathrm{~V} / \mathrm{cm})$ and magnetic $(7 \mathrm{G})$ fields applied along the laser polarization axis. From the time-of-flight and position on the detectors the full momentum vectors of the coincident recoil ions and electrons were calculated. Fluctuations of the laser intensity from pulse to pulse were monitored during the experiment and did not exceed 5\%. Absolute calibration of the peak laser intensity was performed using a clear kink in the measured photoelectron momentum distribution from single ionization of $\mathrm{Ne}$, which corresponds to the maximum drift momentum of $2\left(U_{p}\right)^{1 / 2}$, (where $U_{p}=I / 4 \omega^{2}$ is the ponderomotive potential, $I$ the light intensity and $\omega$ its frequency) that electrons can gain from the laser field ${ }^{16}$. A detailed description of the spectrometer and the coincident measurement scheme, as well as the structure of a typical $\mathrm{H}_{2}$ fragmentation spectrum can be found elsewhere ${ }^{17,18}$.

One of the decisive features of our pump-probe setup is its long-term stability on a sub-fs level. Comparing the autocorrelation pattern obtained from the ion signal with the intensity autocorrelation measured with a photodiode on the laser table before and after the experiment (Fig. 2), we found very similar patterns. This indicates that the optical arrangement was interferometrically stable during the acquisition time of more than 50 hours. Slight differences which are observed in the wings of the autocorrelation spectrum are mainly due to the different intensity dependence of various fragmentation channels. As can be seen from the photodiode data in Fig. 2, in the region where two patterns differ, the molecule is exposed to a non-zero laser field even for the interference minima. The intensity of each laser pulse was $2 * 10^{14} \mathrm{~W} / \mathrm{cm}^{2}$. According to Posthumus et al ${ }^{8}$, the yield of $\mathrm{H}_{2}{ }^{+}$ions reaches its maximum below this value, 


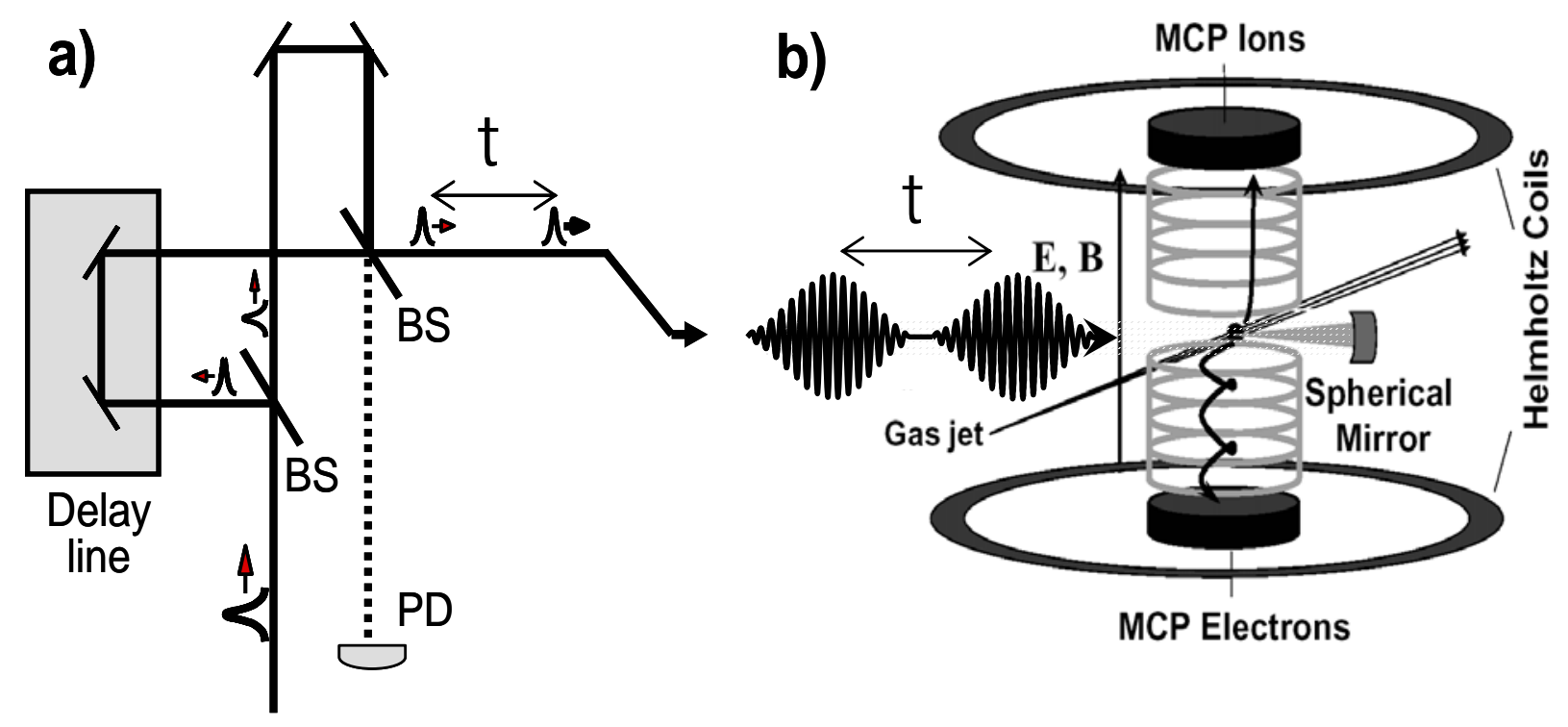

Figure 1. Sketch of the experimental setup. (a) Mach-Zehnder interferometer. BS: beam splitters; PD: photodiode. (b) 'Reaction microscope'.

whereas for higher intensities this channel is depleted via dissociation or/and CE reactions. This can be seen in the inset ofFig. 2, where the autocorrelation pattern obtained from the $\mathrm{H}_{2}^{+}$yield only is shown. Accordingly, the delays for which the $\mathrm{H}_{2}{ }^{+}$ion yield is maximal do not coincide with the maxima of the electric field (see also Section 4), which smears out the wing of the 'in situ' autocorrelation pattern.

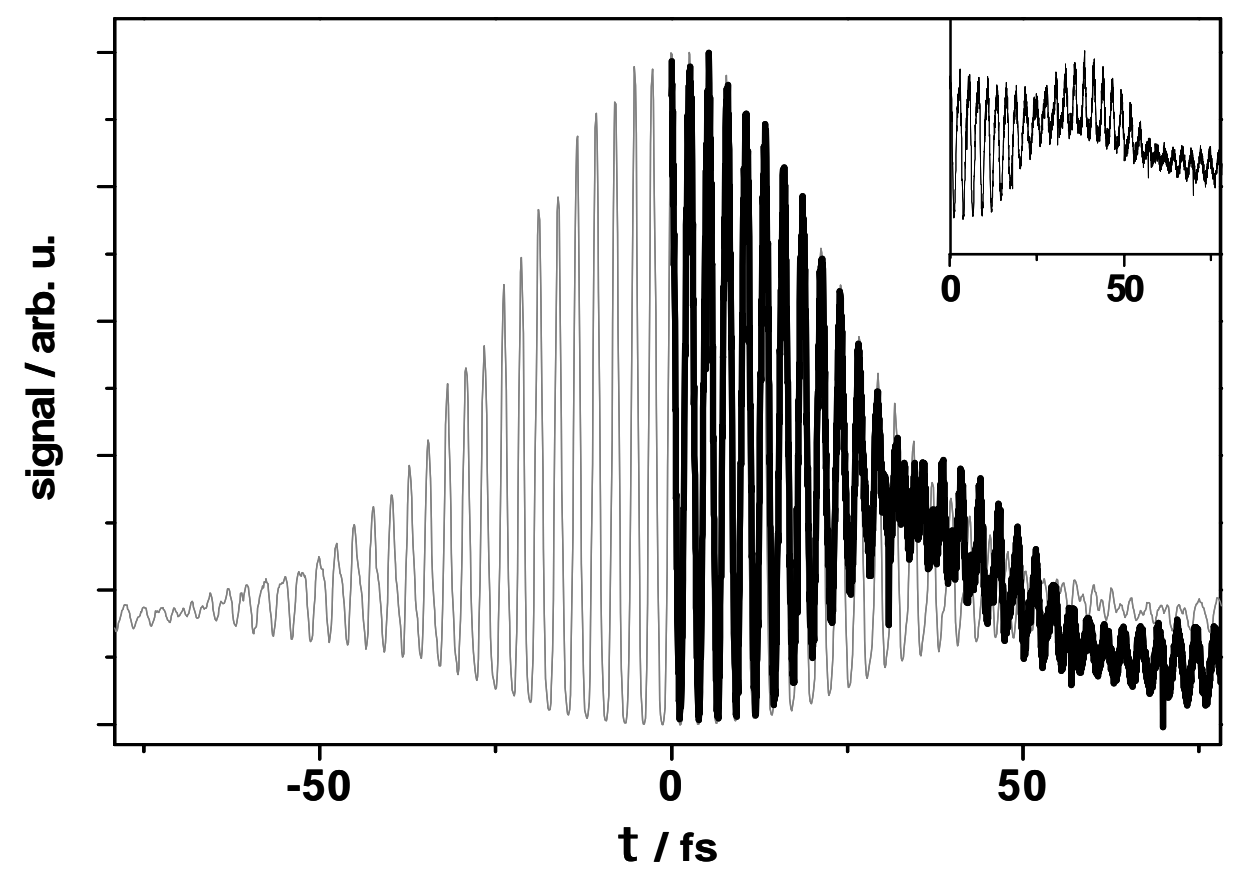

Figure 2. Autocorrelation pattern measured with the photodiode (thin grey line) and obtained from the total ion yield (thick black line). Inset: autocorrelation pattern obtained with $\mathrm{H}_{2}{ }^{+}$ions signal only. 


\section{3. $H_{2}$ FRAGMENTATION CHANNELS IN STRONG LASER FIELDS}

Fig. 3 schematically shows the different fragmentation pathways of $\mathrm{H}_{2}$ in a strong $800 \mathrm{~nm}$ laser field and the relevant potential curve diagram. In single ionization of $\mathrm{H}_{2}$ the initial vibrational ground state is promoted in an almost vertical transition onto the electronic ground state potential curve $1 \mathrm{~s} \sigma_{\mathrm{g}}$ of $\mathrm{H}_{2}{ }^{+}$. Since the wave packet is not an eigenstate anymore it will start to propagate on the new potential curve defining a timescale for the subsequent fragmentation processes. The molecular ion can either dissociate or be further ionized leading to a pair of protons (CE). Up to now there were no experimental evidences found for direct molecular dissociation leading to a pair of neutral hydrogen atoms $^{2}$.

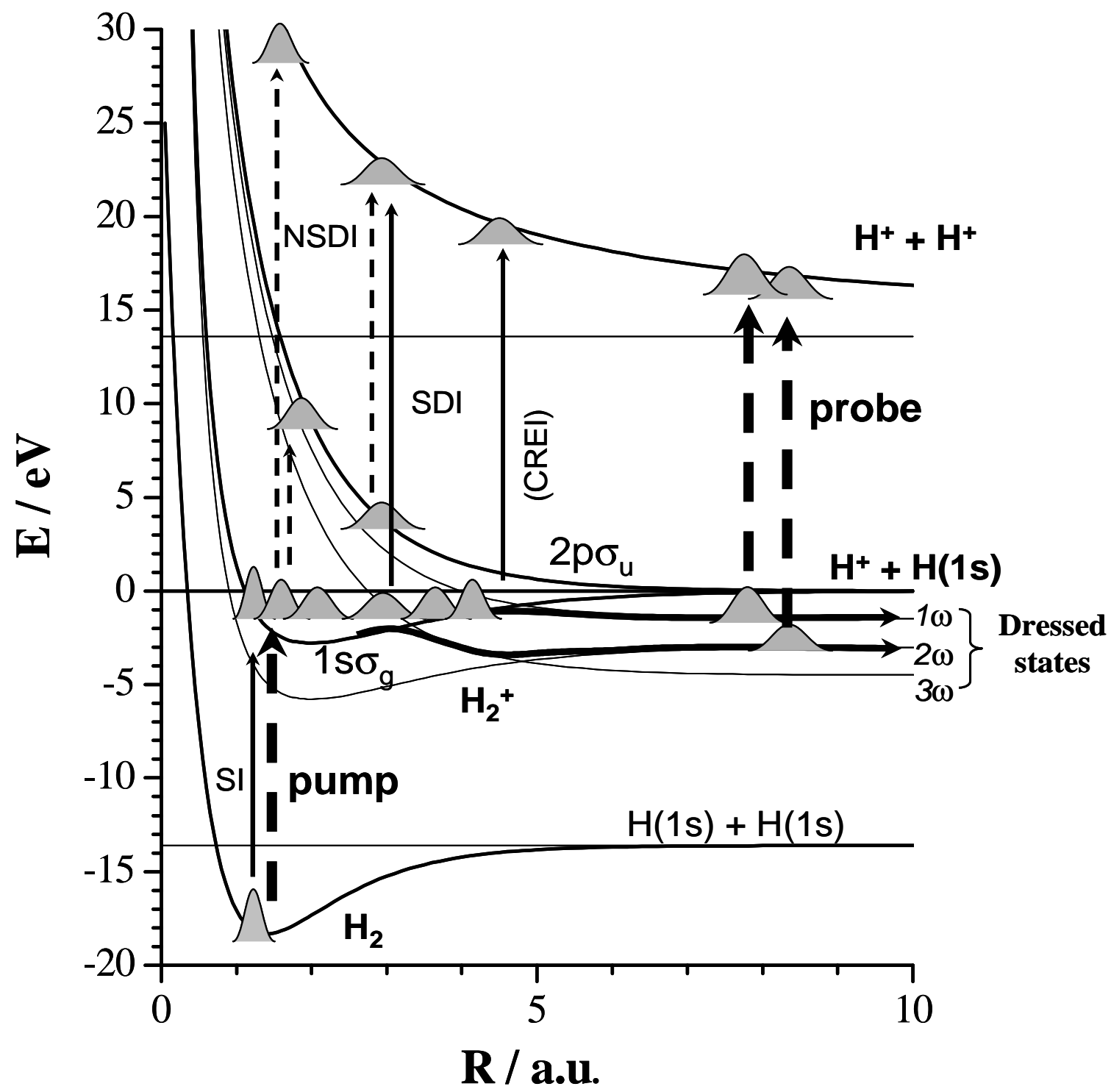

Figure 3. Potential curves of the two lowest electronic states of $\mathrm{H}_{2}+$ dressed with $\mathrm{n}(\mathrm{n}=1,2,3)$ photons. The arrows indicate different fragmentation pathways. SI: single ínization; SDI: sequential double ionization; NSDI: non-s sequential double íonization; CREI: charge resonance enhanced ionization. 


\subsection{Dissociation}

Dissociation is driven by a strong dipole interaction of the two lowest electronic states $1 \mathrm{~s} \sigma_{\mathrm{g}}$ and $2 \mathrm{p} \sigma_{\mathrm{u}}$ in the presence of a strong field along the molecular axis. In a cycle-averaged Floquet description ${ }^{19,20}$ one finds a series of dressed potential curves spaced by the photon energy. Via avoided crossings the molecular ion can dissociate by a net absorption of one or two photons ( $1 \omega$ and $2 \omega$ channels, respectively). In the latter case, absorption of three photons in the first avoided crossing is followed by a re-emission of one photon at the second crossing. Since for each photon the parity of the molecular state must change, there is no direct two-photon process. In the fragment kinetic energy spectrum these channels manifest themselves as two peaks below $1 \mathrm{eV}$ per proton. A comprehensive discussion on the dynamics and the interplay of different dissociation mechanisms referred to as "bond softening"21, vibrational trapping, or "bond-hardening",22, "above-threshold"23, "below-threshold"24 or "zero-photon" (Posthumus et al 2000) dissociation can be found in the reviews by Giusti-Suzor et $\mathrm{al}^{1}$ and Posthumus ${ }^{2}$.

\subsection{Double ionisation $(\mathrm{CE})$}

Similar to the case of atoms, double ionization of molecules in strong laser fields can occur via sequential or nonsequential pathways. The former mechanism becomes more complex in the molecular case since the ionisation potential of the molecular ion depends on the internuclear separation $R$. In addition, at intermediate $R$ an even stronger enhancement of the field ionisation rates is observed due to the interaction of the $1 \mathrm{~s} \sigma_{\mathrm{g}}$ and $2 \mathrm{p} \sigma_{\mathrm{u}}$ states, which causes an oscillating localisation of the electron cloud at one of the nuclei. This effect of charge resonance enhanced ionisation $(\mathrm{CREI})^{12-15}$ gives the highest ionisation probabilities for $\mathrm{H}_{2}^{+}$in a range of internuclear distance from 4 to 7 a.u. and $9-12$ a.u., corresponding to a KER per proton of 2 to $4 \mathrm{eV}$ and $1-1.5 \mathrm{eV}$, respectively. CREI was found to be a dominant sequential double ionization channel in most of the measurements on $\mathrm{H}_{2}$ and $\mathrm{D}_{2}{ }^{8,9,22,26-28}$. However, only the first (highenergy) region of enhanced ionization was usually observed, whereas the first indication on the existence of low-energy $\mathrm{CE}$ structures were found only very recently ${ }^{18,29}$. Since the dipole interaction occurs along the molecular axis, the corresponding Coulomb explosion contribution is strongly peaked along the laser polarization direction ${ }^{2}$. The results of recent experiments ${ }^{18,30,31}$ have shown that if intense $\left(\sim 10^{15} \mathrm{~W} / \mathrm{cm}^{2}\right)$ few-cycle laser pulses are used, the "atomic-like" sequential double ionization, which results in more energetic Coulomb explosion fragments with almost isotropic angular distribution, starts to compete with CREI. These two channels were referred to as "sequential ionization" and "enhanced ionization" "30,31. This should not lead to confusion: both processes are definitely sequential and the essential difference is the extreme R-dependence of the "enhanced ionization" (CREI).

In the case of a linearly polarized laser field the first emitted electron can be driven back and recollide after about three quarters of an optical cycle with its parent ion. The returning electron may either kick out directly the second electron through recollision-ionization or promote the bound electron into an excited state, which can then be easily fieldionized. For $\mathrm{H}_{2}$ the non-sequential double ionization channel has been identified as a high-energy shoulder in the fragment energy spectra for linear polarization, which disappears for circular polarization, since in this case the electron does not recollide with its parent ion ${ }^{30-32}$. Non-sequential double ionization leads to the largest fragment energies since it is a fast process, which for the first electron return gives the nuclei less than one optical cycle to separate.

The probability of "atomic-like" sequential double ionization becomes non-negligible only for few-cycle pulses and very high intensities. On the other hand, recollision-induced ionization occurs on a very short time scale and is, therefore, a single pulse phenomenon. Thus, the main double ionization mechanism addressed in the present work is CREI at relatively large internuclear distances.

\section{RESULTS AND DISCUSSION}

In Fig. 4 the kinetic energy of protons created by $\mathrm{CE}$ of $\mathrm{H}_{2}$ molecules is plotted as a function of the delay between the two 25 fs laser pulses. In order to separate CE events from dissociation fragments, only those events are shown where two protons have been detected in coincidence which in addition fulfill the momentum conservation condition. The intensity of each pulse was $2 * 10^{14} \mathrm{~W} / \mathrm{cm}^{2}$. The count rate for non-overlapping pulses was set to $\sim 0.05$ ions per laser shot leading to a negligible fraction of false coincidences. For delays close to zero, if the two pulses interfere constructively, the peak intensity is four times higher, resulting in a count rate increase of up to 0.5 per shot and, thus, in a noticeable amount of false coincidences in the low-energy region ${ }^{18}$. 


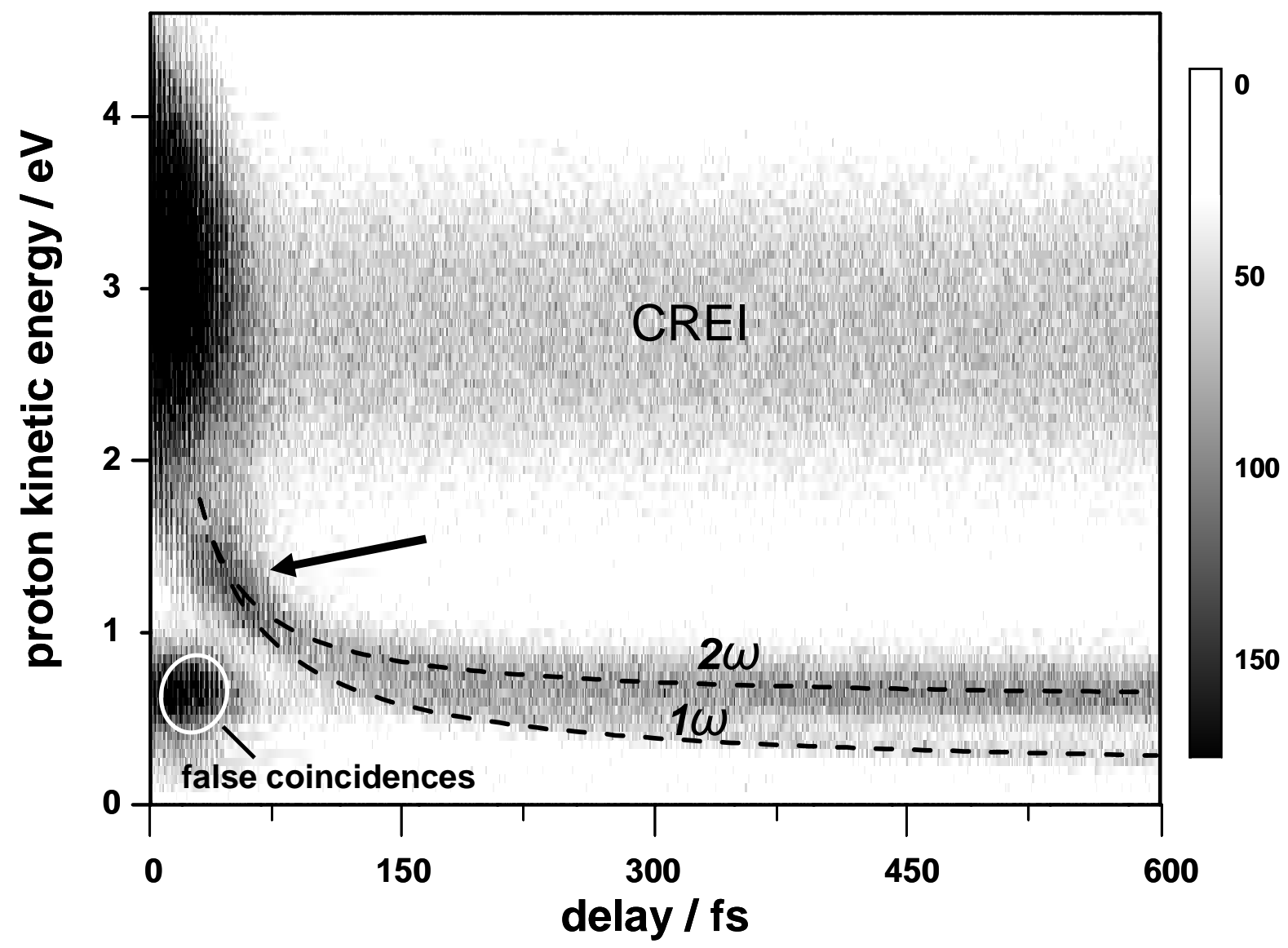

Figure 4. The measured kinetic energy of protons created by $\mathrm{CE}$ of $\mathrm{H}_{2}$ as a function of the delay between two identical 25 fs laser pulses. Dashed lines: classically simulated wave packet propagation for dissociation of $\mathrm{H}_{2}^{+}$via $1 \omega$ and $2 \omega$ channels. The arrow indicates an enhancement corresponding to the maximum of the $\mathrm{H}_{2}{ }^{+}$ionization rate about $\mathrm{R}=10$ a.u..

The spectrum of "true" coincidences in Fig. 4 can be separated into two parts, with proton energies above and below 2 $\mathrm{eV}$, respectively. Outside the region where the two pulses overlap the high-energy band is delay-independent. This structure was observed in numerous previous studies ${ }^{8,9,18,22,26-32}$ and originates from enhanced ionization of the $\mathrm{H}_{2}^{+}$ molecular ion at internuclear distances $\mathrm{R}$ of $\sim$ 4-7 a.u., as was discussed above. Higher proton energies which can be observed in the region of overlapping pulses are due to the higher laser intensity experienced by the molecule: it was demonstrated that with increasing intensity the CREI peak broadens and shifts to higher energies ${ }^{18,26-28}$.

Here, we concentrate on the region below $2 \mathrm{eV}$, where a clear delay-dependent structure appears, which is not observed with a single pulse. Starting from the same energy region as the CREI peak, it propagates towards lower and lower energies with increasing delay. Finally, for delays larger than $300 \mathrm{fs}$, it evolves into two clearly separated bands. This time-dependent structure originates from the process, where the first (pump) pulse ionizes the neutral molecule and induces the dissociation of the molecular ion via Floquet one- $(1 \omega)$ or two-photon $(2 \omega)$ channels. Depending on the delay of the subsequent probe pulse, which ionizes the dissociating $\mathrm{H}_{2}{ }^{+}$ion at different $\mathrm{R}$, different Coulomb explosion energies of the protons are observed. The measured kinetic energy then consists of the Coulomb energy $\left(E_{C}=1 / R\right)$ and the kinetic energy the proton acquired in the dissociation process. 
At very large delays the Coulomb repulsion becomes negligible and the energy distribution of the protons from postdissociative ionization approaches that for the dissociation by a single pulse. Under present conditions, the single pulse spectrum exhibits two maxima at $0.3 \mathrm{eV}$ for the $1 \omega$ and at $0.65 \mathrm{eV}$ for the $2 \omega$ channel, respectively. Using these asymptotic values and assuming a classical motion of the protons, we reconstructed the propagation of the dissociating wave packets (dashed lines in Fig. 4). Initial conditions, i.e. $R_{1 \omega, 2 \omega}(\tau=100 \mathrm{fs}$ ) were chosen in order to fit the experimental data. The good agreement with the experimental data demonstrates that time-resolved CE imaging indeed provides a direct way to map moving nuclear wave packets ${ }^{33-35}$.

The delay-dependent spectrum of the post-dissociative ionization exhibits a noticeable enhancement in the region of 1$1.5 \mathrm{eV}$ kinetic energies (indicated by the arrow in Fig. 4). It might most likely be explained by considering the Rdependence of the ionization rate of dissociating $\mathrm{H}_{2}^{+}$molecular ions. Several theoretical studies ${ }^{12-15}$ have predicted the existence of a second maximum in the ionization probability around $\mathrm{R}=10$ a.u. $\left(\mathrm{E}_{\mathrm{C}}=1.36 \mathrm{eV}\right.$ per proton), well beyond the region corresponding to the CREI-peak observed in single pulse measurements $(2-4 \mathrm{eV})$. The enhancement observed in Fig. 4 reflects this second maximum. However, one has to note that in order to extract the R-dependence explicitly, it is necessary to clearly disentangle it from the wave packet propagation in the corresponding region of internuclear distances.

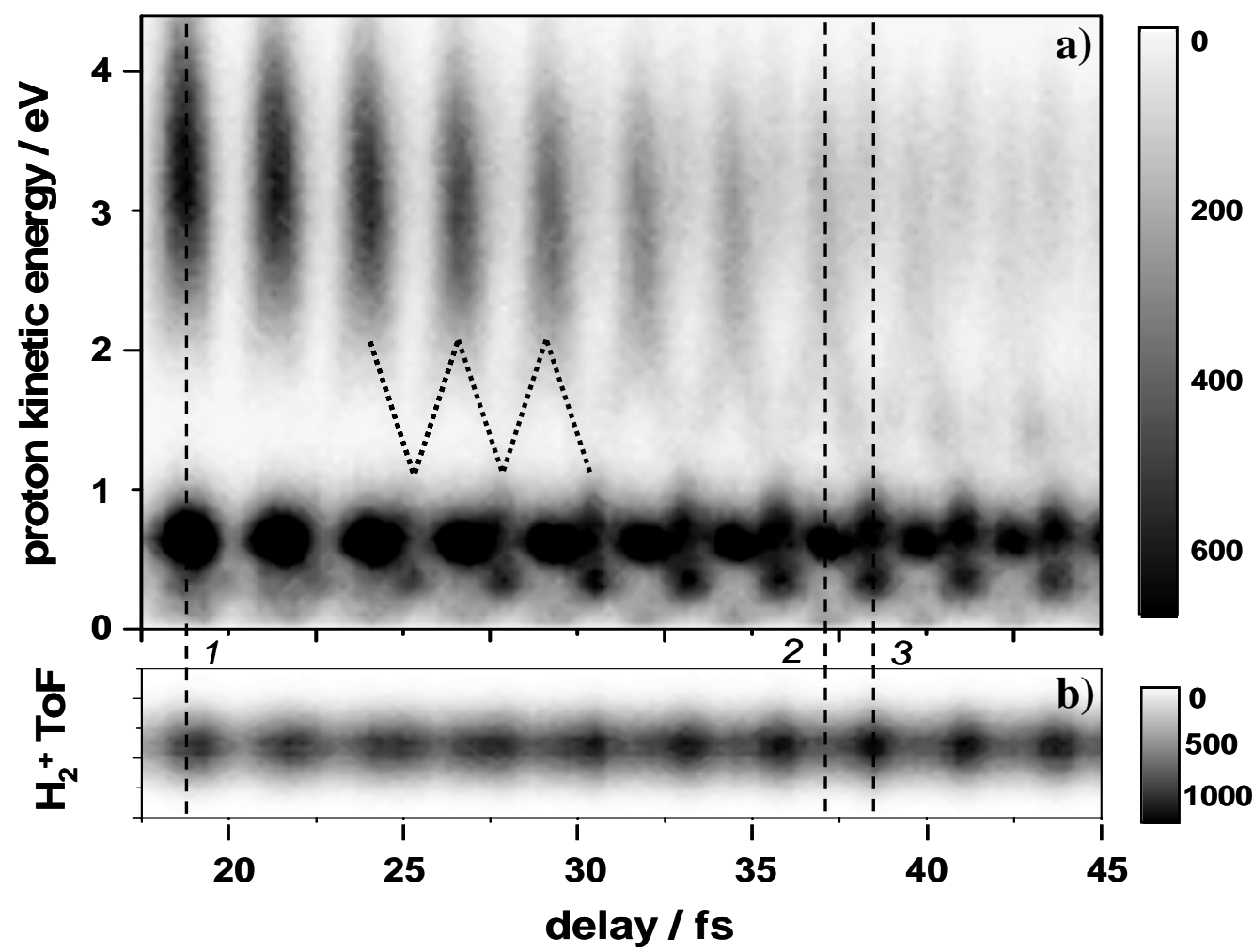

Figure 5. Non-coincident proton kinetic energy spectrum (a) and $\mathrm{H}_{2}{ }^{+}$signal (b) for the delays between 17.5 and 45 fs. The lines 1, 2 and 3 denote delays corresponding to 7, 14 and 14.5 optical cycles, respectively. The dotted line indicates a part of the zigzag structure discussed in text.

In Fig. 5a we present the non-coincident proton kinetic energy spectrum for delays between 17,5 and 45 fs, i.e. for partly overlapping pulses, whereas Fig. 5b displays the time-of-flight signal of $\mathrm{H}_{2}{ }^{+}$ions for the same delays. In contrast to the coincident data of Fig. 4, this spectrum includes both, dissociation and CE events. Protons having energies below $1.2 \mathrm{eV}$ originate from the dissociation of $\mathrm{H}_{2}{ }^{+}$, whereas fragments with higher kinetic energies are due to the CE channels discussed above. The spectrum exhibits the autocorrelation pattern which considerably changes with increasing delay. Its very left part is quite similar to the case of zero delay, where the constructive interference of two 
pulses results in a single pulse of the same duration but with four times increased intensity, and the destructive one leads to almost complete suppression of the pulse. Accordingly, we observe maxima for all fragmentation channels for the former case (see line 1 in Fig. 5) and almost no counts for the latter. However, for larger delays an increasingly large amount of counts appears as well for destructive interference (line 3), and the corresponding kinetic energy spectrum is clearly different from the constructive situation. One can see that in this delay region the maxima of $\mathrm{H}_{2}{ }^{+}$signal are shifted relative to the maxima of the interference pattern, as discussed in Section 2. In addition, a characteristic delaydependent 'zigzag' structure can be observed for proton energies between 1 and $2 \mathrm{eV}$.

In order to consider the fragmentation mechanisms in more detail, we display in Fig. 6 the calculated envelopes of the laser intensity profile for the cases of constructive and destructive interference between the two pulses, (corresponding to the lines 2 and 3 in Fig. 5, respectively). As can be seen, in this delay region the molecule is exposed to a relatively intense laser field for both, constructive as well as destructive interference, however, with very different intensity distributions. Whereas constructive interference results in one long pulse with two slight maxima, the destructive case generates two well separated pulses of lower intensity. Thus, the destructive interference resembles a clean pump probe scheme, and therefore, we can follow the wave packet dynamics even if two pulses overlap.

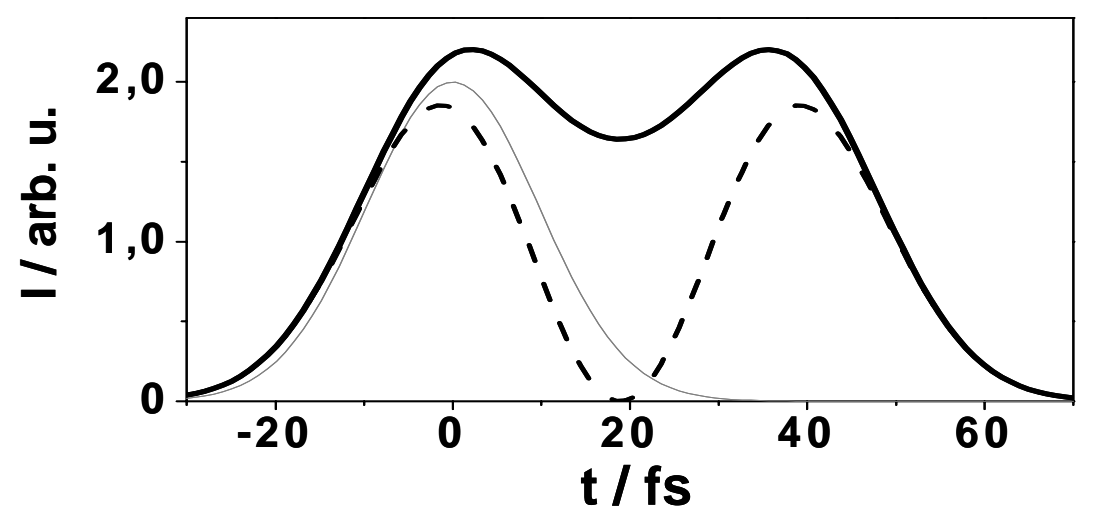

Figure 6. Time dependent laser intensity profile for a single pulse (thin grey), and for two pulses separated by the delay of 14 (solid black, line 2 in Fig. 2) and 14.5 (dashed, line 3 in Fig. 2) optical cycles, i.e. for constructive and destructive interference, respectively. The intensity of a single pulse is $2 * 10^{14} \mathrm{~W} / \mathrm{cm}^{2}$.

Fig. 7 displays total (a) and coincident (b) proton kinetic energy spectra corresponding to the laser intensity profiles shown in Fig. 6. The coincident spectra (Fig. 7b), where only the CE events are included, manifest a clear splitting of the CE peak for destructive interference. For the constructive case (open circles in Fig. 7b) the second ionization can occur for all time intervals $\tau_{12}$ after the removal of the first electron until the pulse falls down, thus, covering the whole range of Coulomb energies from 1 to $4 \mathrm{eV}$ ( $\tau_{12}$ denotes the time between the first and second ionization step). For the destructive interference, instead, the second step can occur either within the same pulse (first or second), or upon the arrival of the second pulse. This leads to a CE spectrum with two maxima around 3 and $1.5 \mathrm{eV}$, respectively (full circles in Fig 7b). The maximum at $3 \mathrm{eV}$ is formed if both ionization steps occur within the same pulse and is indeed very close to the true single-pulse case (thin line), whereas the peak around $1.5 \mathrm{eV}$ corresponds to the situation where the second pulse ionizes the dissociating $\mathrm{H}_{2}{ }^{+}$ion, created by the first one. For two well separated pulses (i.e., destructive interference, dashed line in Fig. 6) certain values of $\tau_{12}$ can not contribute, and thus, CE events with energies of about 2 $\mathrm{eV}$, corresponding to these $\tau_{12}$, are missing. The 'zigzag' structure observed in Fig. 5 reflects the molecular response on the changing field configurations, corresponding to all intermediate situations, from two well separated pulses to one long pulse.

Considering Fig. 7, one can also understand why an additional enhanced ionization maximum, which can be observed in Fig. 4, was not resolved in numerous previous experiments on $\mathrm{H}_{2}\left(\mathrm{D}_{2}\right)$. In a single pulse measurement the laser pulse should be long enough for a molecule to reach the corresponding critical internuclear distances $(\sim 10$ a.u. $)$. However, in this case a considerable part of the wave packet will be ionized while passing the first maximum of enhanced ionization 


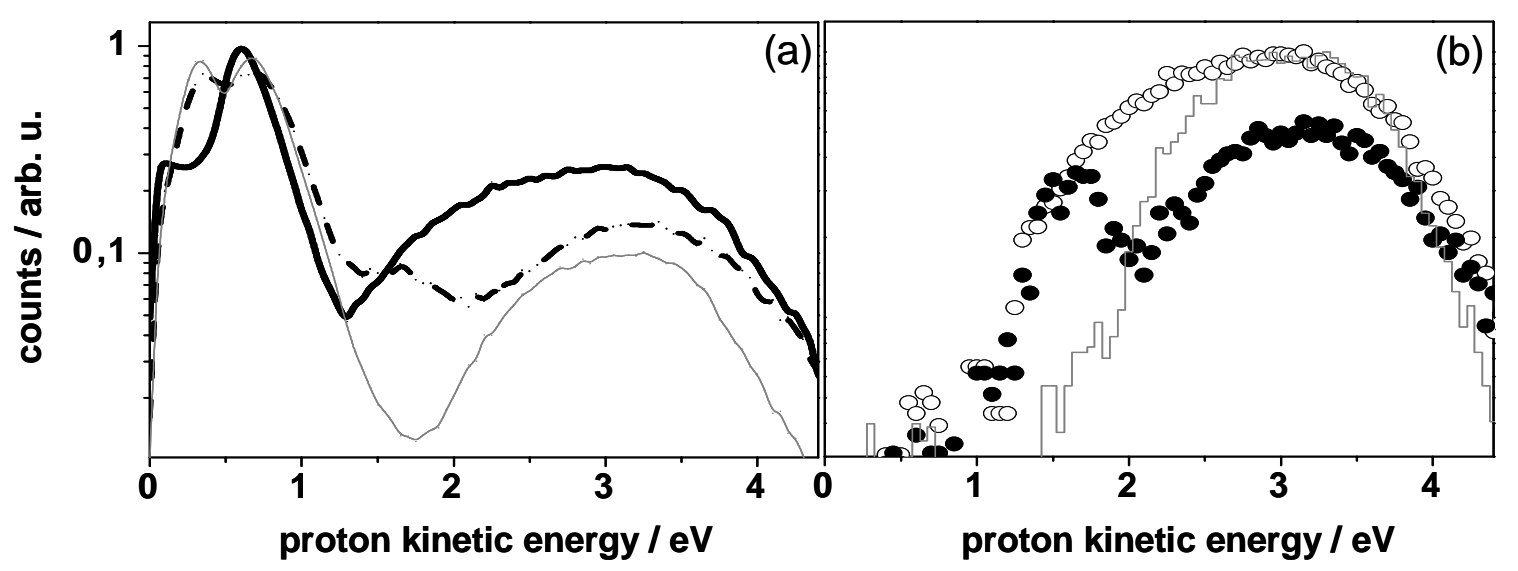

Figure 7. Total (a) and coincident (b) proton kinetic energy spectra corresponding to the laser intensity profiles shown in Fig. 6. (a) Thin grey line: single pulse. Solid black line: delay of 14 optical cycles, constructive interference. Dashed line: delay of 14.5 optical cycles, destructive interference. (b) Thin grey line: single pulse. Open symbols: delay of 14 optical cycles, constructive interference. Full symbols: delay of 14.5 optical cycles, destructive interference. The two-pulse data were integrated over the delay region $\pm 0.3 \mathrm{fs}$ around lines 2 and 3 of Fig. 5 for constructive and destructive case, respectively. Thus, the relative height of these two curves reflects the ionization probabilities for corresponding field configurations, whereas the single-pulse data were arbitrarily normalized for visual convenience.

( $\mathrm{R} \sim 4-7$ a.u.), similar to the constructive case in Fig. 7b. Thus, the second maximum manifests itself in the spectrum only if one uses two well-separated pulses which are short enough. This situation was partly realized in our recent study on $\mathrm{H}_{2}$ fragmentation by few-cycle pulses ${ }^{18}$ where first indications on the existence of the low-energy CE peak have been obtained. However, due to uncertainties in the pulse shape a definite conclusion could not be drawn.

For the delay region shown in Fig. 5 not only the CE but also the dissociation dynamics differs strongly for the cases of constructive and destructive interferences between the two pulses. For the line 3 in Fig. 5 (destructive interference), the low-energy part of the spectrum (up to $1.2 \mathrm{eV}$ ) is similar to that obtained with a single pulse (see Fig. 7a), indicating that in this case both pulses induce the dissociation independently. For the other case (line 2 in Fig. 5, solid line in Fig. 6 ) the $1 \omega$ dissociation peak is suppressed and shifted towards zero energy, whereas the $2 \omega$ peak, being also slightly shifted towards lower energies, is considerably enhanced. This change of the branching ratio is due the differences in the peak intensity and the effective pulse duration (see Fig. 6). This intensity dependence was already observed experimentally ${ }^{32}$ : the probability of the three-photon transition required for the $2 \omega$ channel grows with increasing intensity faster than that of the one-photon process, and, since the $\mathrm{H}_{2}{ }^{+}$ion first passes the three-photon crossing, this results in an enhancement of the $2 \omega$ in comparison with the $1 \omega$ channel. The effect is further enhanced since for the case of constructive interference the molecule experiences an effectively longer pulse. It was shown that the $2 \omega$ channel has higher relative weight for longer pulse durations ${ }^{18}$.

The shift of the $1 \omega$ and $2 \omega$ peaks towards lower energies is also a consequence of different pulse shapes ${ }^{22,28}$. Frasinski et $a l .^{22}$ observed that the $1 \omega$ peak shifts to almost zero energy with increasing pulse duration. This effect was attributed to the pulse-length dependent decay dynamics of the so-called 'vibrationally trapped', or 'bond hardened' states. Due to the more efficient trapping for the case of constructive interference we observe a similar shift of the $1 \omega$ peak. The origin of the slight shift of the $2 \omega$ peak is not yet completely clear. The most likely reason is the difference in the distribution of contributing vibrational states, which depends on both, intensity ${ }^{36}$ and pulse duration.

\section{CONCLUSIONS AND OUTLOOK}

We have shown that time-resolved CE imaging with two 25 fs laser pulses enables a direct mapping of the dissociating nuclear wave packets of $\mathrm{H}_{2}{ }^{+}$molecules. In contrast to earlier pump-probe studies on $\mathrm{H}_{2}{ }^{8}$ and $\mathrm{D}_{2}{ }^{9}$, we were able to 
separate the motion of the wave packets dissociating via one- and two-photon Floquet channels. We have demonstrated that our interferometrically stable setup, where the delay between two pulses can be scanned with sub-fs resolution, allows us to extend the pump-probe scheme into the region of partly overlapping pulses, selecting delays for which the pulses interfere destructively. Moreover, we have shown that changing the electric field profile in a controlled way, one can manipulate the ultrafast molecular fragmentation dynamics, suppressing and enhancing certain fragmentation channels. Vice versa, the possibility to observe an autocorrelation pattern with different fragmentation channels, and thus, with different degree of nonlinearity, opens new ways for 'in situ' diagnostics of the laser pulses itself, which is of crucial importance for experiments with few-cycle pulses.

In the coincident delay-dependent kinetic energy spectrum we have observed a signature of an additional enhanced ionization maximum in R-dependence of the ionization probability for $\mathrm{H}_{2}{ }^{+}$. However, one has to note that in order to extract the R-dependence explicitly, it is necessary to clearly disentangle it from the wave packet propagation in the corresponding region of internuclear distances. This can be realized within pump-probe experiments using considerably shorter laser pulses which should be performed in two modes: one, using an extremely intense probe pulse in order to map the time development of the nuclear wave packet, as suggested by Chelkovski et. al ${ }^{34}$, and another one, with a probe pulse of moderate intensity. Such combined analyses will allow a direct comparison of the experimental data with theoretically calculated R-dependent rates. Corresponding experiments with 6-7 fs laser pulses are currently underway.

\section{ACKNOWLEDGEMENTS}

The authors are grateful to M. Lein and A. Saenz for the numerous fruitful discussions.

\section{REFERENCES}

1 A. Giusti-Suzor, F.H. Mies, L.F. DiMauro, E. Charron and B. Yang J. Phys. B 28309 (1995).

2 J.H. Posthumus, Rep. Prog. Phys. 67623 (2004).

3 A.H. Zewail, J. Phys. Chem. 1045660 (2000).

4 Z. Vager, R. Naaman and E.P. Kanter, Science 244426 (1989).

5 Z. Vager, T. Graber, E.P. Kanter and D. Zajfman, Phys. Rev. Lett. 703549 (1993).

${ }^{6}$ H. Stapelfeldt, E. Constant and P.B. Corkum, Phys. Rev. Lett. 743780 (1995).

7 H. Stapelfeldt, E. Constant, H. Sakai and P.B. Corkum, Phys. Rev. A 58426 (1998).

8 J.H. Posthumus et al, J. Phys. B 32 L93 (1999).

9 C. Trump, H. Rottke and W. Sandner, Phys. Rev. A 592858 (1999).

${ }^{10}$ H. Niikura et al, Nature 417917 (2002).

${ }^{11}$ H. Niikura et al, Nature 421826 (2003).

${ }^{12}$ T. Zuo and A.D. Bandrauk Phys. Rev. A 52 R2511 (1995).

${ }^{13}$ M. Plummer and J.F. McCann J. Phys. B 294625 (1996).

${ }^{14}$ L.-Y. Peng, D. Dundas, J.F.McCann, K.T. Taylor and I.D. Williams, J. Phys. B 36 L295 (2003).

${ }^{15}$ M. Vafaee and H. Sabzyan, J. Phys. B 374143 (2004).

${ }^{16}$ V.L.B. de Jesus et al, J. Phys. B 37, L161 (2004).

${ }^{17}$ V.L.B. de Jesus et al., J. Electron Spectrosc. Relat. Phenom., 141127 (2004).

${ }^{18}$ A. Rudenko et al., J. Phys. B 38, 487 (2005).

${ }^{19}$ J.H. Shirley, Phys. Rev. 138 B979 (1965).

${ }^{20}$ A.D. Bandrauk and M.L. Sink, J. Chem. Phys. 741110 (1981).

${ }^{21}$ P.H. Bucksbaum, A. Zavriyev, H.G. Muller and D.W. Schumacher, Phys. Rev. Lett. 641883 (1990).

${ }^{22}$ L.J. Frasinski et al., Phys. Rev. Lett. 833625 (1999).

${ }^{23}$ A. Giusti-Suzor, X. He, O. Atabek and F.H. Mies Phys. Rev. Lett. 64515 (1990).

${ }^{24}$ R. Numico, A. Keller and O. Atabek Phys. Rev. A 56772 (1997).

${ }^{25}$ J.H. Posthumus et al., J. Phys. B 33 L563 (2000).

${ }^{26}$ T.D G Walsh, F.A. Ilkow and S.I. Chin, J. Phys. B 302167 (1997).

${ }^{27}$ M.R. Thomson et al, J. Phys. B 305755 (1997).

${ }^{28}$ C. Trump et al., Phys. Rev. A 62063402 (2000). 
${ }^{29}$ D. Pavicic, A. Kiess, T.W. Hänsch and H. Figger, Phys. Rev. Lett. 94163002 (2005).

${ }^{30}$ F. Legare et al., Phys. Rev. Lett. 91, 093002 (2003).

${ }^{31}$ A.S. Alnaser et al., Phys. Rev. Lett. 93183202 (2004).

32 A. Staudte et al., Phys. Rev. A 65020703 (R) (2002).

${ }^{33}$ S. Chelkowski, P.B. Corkum and A.D. Bandrauk, Phys. Rev.Lett. 823416 (1999).

${ }^{34}$ S. Chelkowski and A.D. Bandrauk, Phys. Rev. A 65023403 (2002).

${ }^{35}$ B. Feuerstein and U. Thumm, Phys Rev. A 67063408 (2003).

${ }^{36}$ X. Urbain et al., Phys. Rev. Lett. 92163004 (2004). 